\title{
Effect of substrate on optical bound states in the continuum in 1D photonic structures
}

Sadrieva, Z. F.; Sinev, I. S.; Samusev, A. K.; lorsh, I. V.; Koshelev, K. L.; Takayama, Osamu; Malureanu, Radu; Lavrinenko, A. V.; Bogdanov, A. A.

Published in:

AIP Conference Proceedings

Link to article, DOI:

$10.1063 / 1.4998034$

Publication date:

2017

Document Version

Publisher's PDF, also known as Version of record

Link back to DTU Orbit

Citation (APA):

Sadrieva, Z. F., Sinev, I. S., Samusev, A. K., lorsh, I. V., Koshelev, K. L., Takayama, O., Malureanu, R., Lavrinenko, A. V., \& Bogdanov, A. A. (2017). Effect of substrate on optical bound states in the continuum in 1D photonic structures. AIP Conference Proceedings, 1874, [030005]. https://doi.org/10.1063/1.4998034

\section{General rights}

Copyright and moral rights for the publications made accessible in the public portal are retained by the authors and/or other copyright owners and it is a condition of accessing publications that users recognise and abide by the legal requirements associated with these rights.

- Users may download and print one copy of any publication from the public portal for the purpose of private study or research.

- You may not further distribute the material or use it for any profit-making activity or commercial gain

- You may freely distribute the URL identifying the publication in the public portal 


\section{Effect of substrate on optical bound states in the continuum in 1D photonic structures}

Z. F. Sadrieva, I. S. Sinev, A. K. Samusev, I. V. Iorsh, K. L. Koshelev, O. Takayama, R. Malureanu, A. V. Lavrinenko, and A. A. Bogdanov

Citation: AIP Conference Proceedings 1874, 030005 (2017);

View online: https://doi.org/10.1063/1.4998034

View Table of Contents: http://aip.scitation.org/toc/apc/1874/1

Published by the American Institute of Physics

\section{Articles you may be interested in}

Retrieval procedure of effective conductivity for plasmonic resonant anisotropic metasurface AIP Conference Proceedings 1874, 030040 (2017); 10.1063/1.4998069

Advanced fabrication of hyperbolic metamaterials

AIP Conference Proceedings 1874, 020004 (2017); 10.1063/1.4998025

Photoluminescence behavior of nanoimprinted halide perovskite at low temperatures

AIP Conference Proceedings 1874, 030029 (2017); 10.1063/1.4998058

Theory of Fano resonance for scattering spectrum of all-dielectric spherical resonators

AIP Conference Proceedings 1874, 030020 (2017); 10.1063/1.4998049

Spectroscopy of topological photonic states in dielectric metasurfaces

AIP Conference Proceedings 1874, 030014 (2017); 10.1063/1.4998043

Strong twisting of light with hyperbolic metasurfaces

AIP Conference Proceedings 1874, 040021 (2017); 10.1063/1.4998094 


\title{
Effect of substrate on optical bound states in the continuum in $1 D$ photonic structures
}

\author{
Z. F. Sadrieva ${ }^{1, a)}$, I. S. Sinev ${ }^{1}$, A. K. Samusev ${ }^{1}$, I. V. Iorsh ${ }^{1}$, K. L. Koshelev ${ }^{1}$, \\ O. Takayama ${ }^{2}$, R. Malureanu ${ }^{2}$, A. V. Lavrinenko ${ }^{1,2}$ and A. A. Bogdanov ${ }^{1}$ \\ ${ }^{1}$ ITMO University, St. Petersburg 197101, Russia \\ ${ }^{2}$ DTU Fotonik, Technical University of Denmark, Kgs. Lyngby, Denmark \\ ${ }^{a)}$ Corresponding author: zfsadrieva@ corp.ifmo.ru
}

\begin{abstract}
Optical bound states in the continuum (BIC) are localized states with energy lying above the light line and having infinite lifetime. Any losses taking place in real systems result in transformation of the bound states into resonant states with finite lifetime. In this work, we analyze properties of BIC in CMOS-compatible one-dimensional photonic structure based on siliconon-insulator wafer at telecommunication wavelengths, where the absorption of silicon is negligible. We reveal that a high-index substrate could destroy both off- $\Gamma$ BIC and in-plane symmetry protected at- $\Gamma$ BIC turning them into resonant states due to leakage into the diffraction channels opening in the substrate.
\end{abstract}

\section{INTRODUCTION}

While localized states with energies under the light line of the surrounding space are ubiquitous [1], optical bound states in the continuum (BICs) are a remarkable exception for this general rule. The BICs are spatially bounded (localized in one, two or even three dimensions [2]) eigenstates of an optical system with quality factor that could, in principle, approach infinity despite lying within the light cone of the surrounding space, i.e. in the continuum spectrum of radiative modes [3]. The concept of BIC was firstly proposed by von Neumann and Wigner in 1929 for electron placed in a specific potential [4]. More recently, another examples of electronic BICs have been predicted theoretically in atomic and molecular systems [5] and in artificial systems [6]. In optics, the term bound state in the continuum first appeared around 2008 [7, 8]. Experimental observation of optical BIC followed only in 2011 [9].

Due to high Q-factor, optical BICs are very promising for many applications ranging from on-chip photonics and optical communications to biological sensing and photovoltaics. It has been already shown [10] that, in nonlinear materials due to the Kerr effect, a robust BIC arises in a self-adaptive way without necessity to tune the material parameters. In theory, BICs have infinite high quality factor, however, for real systems it is limited because of material absorption, technological imperfections, roughness, finite lateral size of samples and leakage into the substrate.

In this work we analyze transformation of BIC into a resonant state due to surface roughness and high-index substrate beneath the structure opening the diffraction channels. We implement a one-dimensional photonic structure based on silicon-on-insulator (SOI) wafer and reveal the critical role of the substrate on BIC. We demonstrate its transformation to a resonant state [11], having a finite radiation lifetime, with the change of the thickness of the silica spacer separating the photonic structure and the substrate of SOI wafer.

\section{SIMULATION AND ANALYSIS}

The design of the photonic structure under study is shown in Fig. 1(a). It consists of rectangular bars made of crystalline silicon surrounded by fused silica. Since the array is periodic in the $x$-direction, the eigenstates of the structure are characterized by the frequency $f$, the wave vector along the bars $k_{y}$ and by the Bloch quasi-wave vector $k_{x}$ restricted to the first Brillouin zone.

Proceedings of International Conference on Metamaterials and Nanophotonics (METANANO-2017)

AIP Conf. Proc. 1874, 030005-1-030005-4; doi: 10.1063/1.4998034

Published by AIP Publishing. 978-0-7354-1554-6/\$30.00 

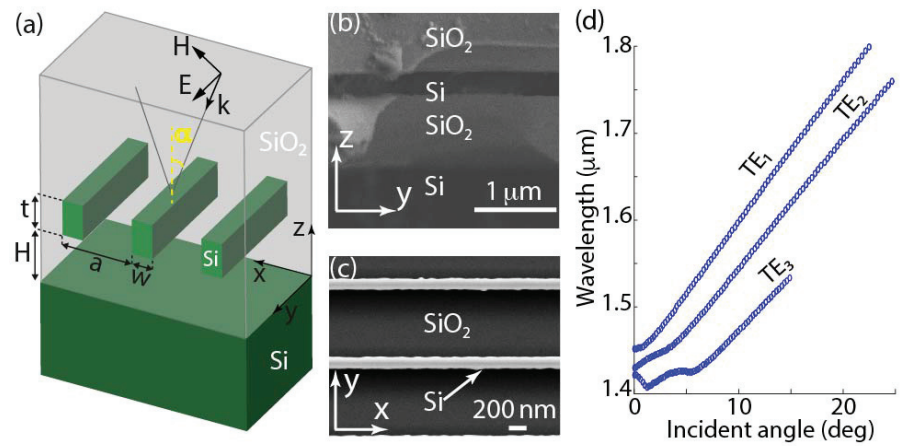

FIGURE 1. (a) Schematic image of photonic structure under consideration. Parameters of the structure: $w=150 \mathrm{~nm} ; a=1000 \mathrm{~nm}$, $t=350 \mathrm{~nm}$. Thickness of the bottom silica layer $H$ is equal to $1 \mu \mathrm{m}$. (b,c) SEM images of the fabricated structure in side and top view, respectively. (d) Calculated dispersion curves for the first three TE modes of the photonic structure.
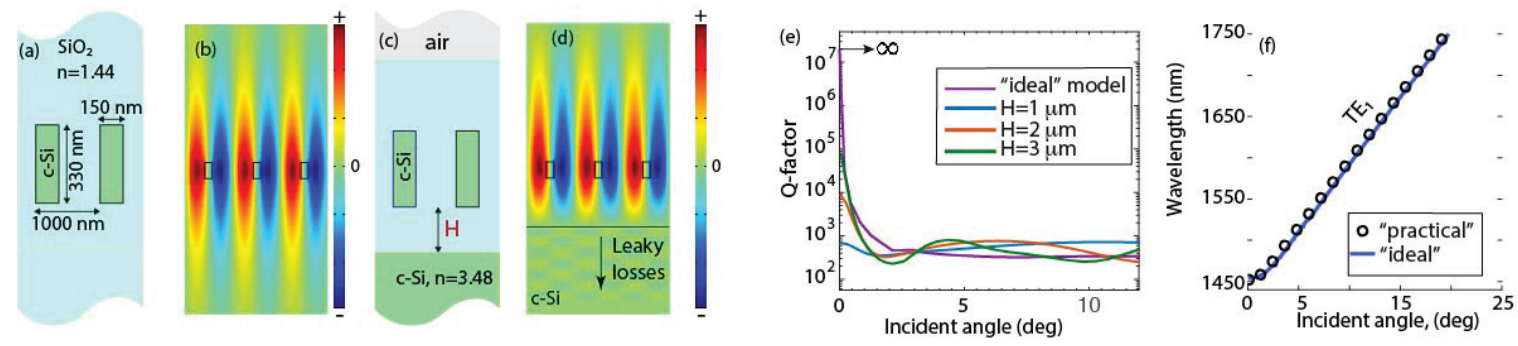

FIGURE 2. Design of the photonic structure in 'ideal' (a) and 'practical' (c) cases. (b) Calculated distribution of $E_{y}$ component for $\mathrm{TE}_{1}$ mode in the 'ideal' case. (d) Calculated distribution of $E_{y}$ component for $\mathrm{TE}_{1}$ mode in the 'practical' case when $H=1 \mu \mathrm{m}$. The TE-polarized excitation wave has normal incidence for both cases. Calculated Q-factor (e) and dispersion curves (f) for both numerical models. Refractive indices of silica and silicon in the considered wavelength range have very weak dispersion [12], so, we put them constants $n_{\mathrm{SiO}_{2}}=1.44$ and $n_{\mathrm{Si}}=3.48$.

We consider two cases: (i) the first one is referred to as 'ideal', because it describes a periodic array of silicon bars and infinite silica medium; (ii) the second one is termed 'practical' and differs from the first case by adding a silicon substrate and surrounding air. The geometries of the first and second cases are shown in Figs. 2(a) and 2(c).

Let us first consider an 'ideal' structure without silicon substrate [see Fig. 2(a)]. The calculated dispersion curves of the three lower TE modes are shown in Fig. 1(d). The modes were numbered in order of occurrence in the spectrum, so the numbers do not indicate the characteristics of electric field. Since the eigenvalue solver returns complex eigenfrequencies $f$, we straightforwardly calculate the radiative Q-factor as

$$
Q=\frac{\operatorname{Re}(f)}{2 \operatorname{Im}(f)} .
$$

One can see from Fig. 2(b,e) that the Q-factor of the $\mathrm{TE}_{1}$ mode tends to infinity at $\Gamma$-point of $\mathbf{k}$-space (normal incidence). In contrast, the $\mathrm{Q}$-factor of the $\mathrm{TM}_{1}$ mode diverges at normal and oblique incidence. At these points, leaky resonances turn into localized eigenmodes that do not decay and light becomes perfectly confined in the slab [see Figs. 2(b)]. It follows from Fig. 2(f) that BICs for the 'ideal' structure appear at a frequency of about $206 \mathrm{THz}$ $(1454 \mathrm{~nm})$ for TE mode, $193 \mathrm{THz}(1550 \mathrm{~nm})$ for TM mode at normal incidence.

In the 'ideal' case (without substrate), all diffraction channels, except zeroth one, are closed since the frequencies 
of at- $\Gamma$ BICs $f_{\Gamma, T E}=206 \mathrm{THz}$ and $f_{\Gamma, T M}=193 \mathrm{THz}$ are below the diffraction limit: $f_{\Gamma}<c /\left(n_{\mathrm{SiO}_{2}} a\right) \approx 207.4 \mathrm{THz}$, where $c$ is the vacuum speed of light. In the 'practical' case, the photonic structure is placed on the silica spacer separating the structure and the silicon substrate. It might seem that the substrate should not destroy the at- $\Gamma$ BIC since it is protected by in-plane symmetry which is not broken by the substrate [13]. However, it is true only for the subwavelength regime when the zeroth diffraction channel is forbidden due to the in-plane symmetry and higher diffraction channels are closed. In our case, the evanescent fields of the at- $\Gamma$ BIC transform in the silicon substrate to propagating (diffractive) waves and the BIC becomes a resonant state. So, for the $\mathrm{TE}_{1}$ mode with $f_{\Gamma}=206 \mathrm{THz}$ we have four waves diffracting at the angles $\alpha_{s}=\arcsin \left[s n_{\mathrm{Si}} c /\left(f_{\Gamma} a\right)\right]$, where $s= \pm 1, \pm 2$. The diffraction angles $\alpha_{s}$ are defined with respect to the $z$-axis. Figure 2(d) shows how the energy stored in BIC leaks into the silicon substrate via open diffraction channels forming the characteristic interference pattern. In contrast, the frequency of at- $\Gamma$ BIC of the $\mathrm{TM}_{1}$ mode lies below the diffraction limit of the silica. Therefore, wave can not radiate into the silica layer and the BIC seemed to be protected by the in-plane symmetry which is not broken by the substrate. However, the volume of the $\mathrm{TM}_{1}$ mode is huge enough to allow the penetration of decaying waves into the silicon substrate, where four additional diffractive channels (with order of $s= \pm 1, \pm 2$ ) into silicon are opened, since the second diffraction limit for the silicon is about $86 \mathrm{THz}$. A more detailed analysis of the structure is represented in Ref. [14].

\section{EXPERIMENT}

To confirm our theoretical results experimentally, we fabricated a grating of silicon nanobars from SOI substrate with $1 \mu \mathrm{m}$-thick silicon oxide layer covered by a 330-nm-thick silicon slab shown in Fig. 1. The grating is fabricated using electron beam lithography. The structure is then covered by $3 \mu \mathrm{m}$ of silicon oxide via sputtering to create symmetric environment for guided modes of the grating.

To characterize the sample, we perform angle-resolved reflectivity measurements using the setup schematically shown in Fig. 3(c). Figure 3(a) shows the measured map of the angle-resolved reflectance spectra of the fabricated structure normalized to the reflectance spectra of the unprocessed SOI wafer. The observed modulation corresponds to the Fabry-Perot modes of the SOI wafer. The interference of the incident light with the leaky modes results in Fano-type resonances [15]. The experimental spectra are in good agreement with the results of the FDTD simulation [Fig. 3(b)]. Comparison of Figs. 1(d) and 3 shows that two the most pronounced lines correspond to $\mathrm{TE}_{1}$ and $\mathrm{TE}_{2}$ modes. As it is shown in the insets of Fig. 3, for small angles of incidence, the Fano-type peak corresponding to the $\mathrm{TE}_{1}$ mode is less pronounced as compared to $\mathrm{TE}_{2}$ mode because of its higher Q-factor.
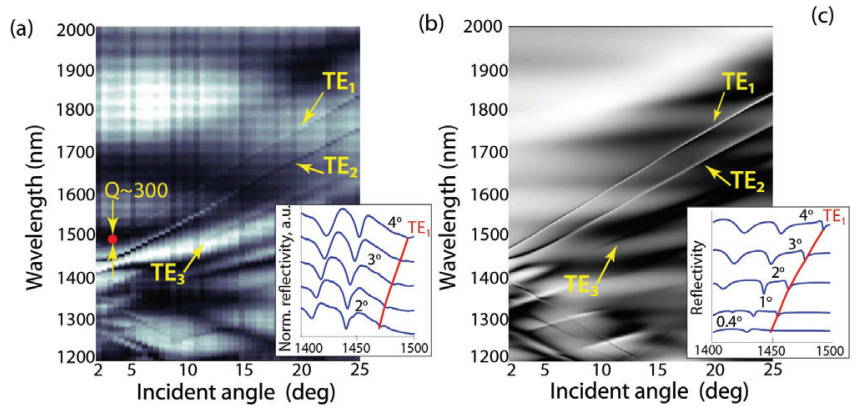

(c)

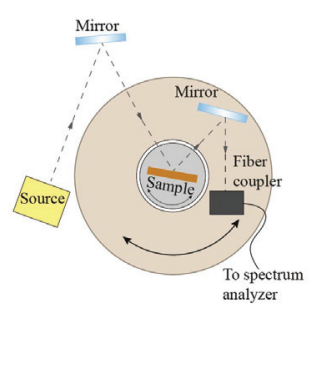

(d)

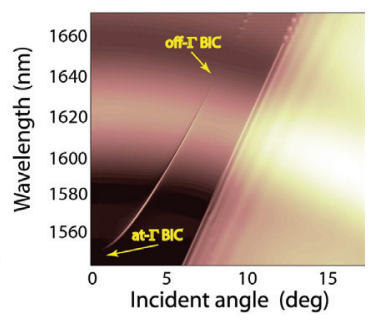

FIGURE 3. (a) Measured reflectivity for TE polarization. The quality factor $Q$ was calculated from experimental results for incident $4^{\circ}$. The inset shows reflection spectra for the angle of incidence from $2^{\circ}$ to $4^{\circ}$. (b) Calculated reflectivity for TE polarization. The inset shows reflection spectra for incidence from $0.4^{\circ}$ to $4^{\circ}$. (c) Scheme of setup for reflectance measurements. (d) Calculated reflectivity for TM mode. Disappearance of the response around $0^{\circ}$ and $8^{\circ}$ indicates a trapped state with no leakage.

The resonance lifetimes can be determined from reflection spectra by extracting the linewidths of the observed Fano features. The results of the extraction for $\mathrm{TE}_{1}$ mode from both experimental and FDTD data are presented in Fig. 4(a) along with the calculated Q-factor from the FEM simulations. Figure 4(a) shows that the Q-factor obtained from the FDTD calculation agrees well with the results of the FEM. The Q-factor extracted from the experimental reflectance spectra, however, reaches slightly lower values than the calculated Q-factor [Fig. 4(a)]. It should be noted that the resolution of the spectrometer allows to measure Q-factors up to $10^{3}$. However, due to divergence of the 
excitation beam in the experiment, we measure the characteristics not of single states with specific Bloch wavenumbers but their convolution. It explains the difference between the experimental data and simulations.



FIGURE 4. (a) Quality factor $Q$ versus incident angle extracted from experimental (shown with dots) and FDTD (dashed line) data and calculated with FEM for 'practical' case (solid line). During both calculation, substrate was taken into account. (b) Quality factor $Q$ versus incident angle for "ideal structure" (without substrate). The data are obtained from eigenfrequency calculation (using FEM) and estimated from calculated reflectance (using FEM).

\section{CONCLUSION}

In summary, we have theoretically and experimentally analyzed properties of the BIC in CMOS-compatible onedimensional photonic structure based on silicon-on-insulator wafer at telecommunication wavelengths. We have revealed that a high-index substrate could destroy even an at- $\Gamma$ BIC protected by the in-plane symmetry and turn it into a resonant state. The obtained results provide useful guidelines for practical implementations of structures supporting optical bound states in the continuum and could find a number of applications in optical communications, on-chip photonics, laser physics and sensing.

\section{ACKNOWLEDGMENTS}

This work is supported by the Russian Science Foundation (17-12-01581).

\section{REFERENCES}

[1] J. D. Joannopoulos, S. G. Johnson, J. N. Winn, and R. D. Meade, Photonic crystals: molding the flow of light, 2nd ed. (Princeton University Press, New Jersey, 2008).

[2] F. Monticone and A. Alù, Phys. Rev. Lett. 112, 1-5 (2014).

[3] C. W. Hsu, B. Zhen, J. Lee, S.-L. Chua, S. G. Johnson, J. D. Joannopoulos, and M. Soljačić, Nature 499, 188-191 (2013).

[4] J. von Neumann and E. P. Wigner, Phys. Zeitschrift 30, 465-467 (1929).

[5] F. H. Stillinger, D. R. Herrick, B. Laboratories, and M. Hill, Phys. Rev. A 11, 446-454 (1975).

[6] E. N. Bulgakov, K. N. Pichugin, A. F. Sadreev, and I. Rotter, JETP Lett. 84, 430-435 (2006).

[7] D. C. Marinica, a. G. Borisov, and S. V. Shabanov, Phys. Rev. Lett. 100, p. 183902 (2008).

[8] E. N. Bulgakov and A. F. Sadreev, Phys. Rev. B 78, p. 75105 (2008).

[9] Y. Plotnik, O. Peleg, F. Dreisow, M. Heinrich, S. Nolte, A. Szameit, and M. Segev, Phys. Rev. Lett. 107, p. 183901 (2011).

[10] E. N. Bulgakov and A. F. Sadreev, Opt. Lett. 39, p. 5212 (2014).

[11] C. W. Hsu, B. Zhen, a. D. Stone, J. D. Joannopoulos, and M. Soljačić, Nat. Rev. Mater. 1, p. 16048 (2016).

[12] M. N. Polyanskiy, Refractive index database, https://refractiveindex.info ().

[13] B. Zhen, C. W. Hsu, L. Lu, a. D. Stone, and M. Soljacic, Phys. Rev. Lett. 113, p. 257401 (2014).

[14] Z. F. Sadrieva, I. S. Sinev, K. L. Koshelev, A. Samusev, I. V. Iorsh, O. Takayama, R. Malureanu, A. A. Bogdanov, and A. V. Lavrinenko, ACS Photonics 4, 723-727 (2017).

[15] S. Fan and J. Joannopoulos, Phys. Rev. B 65, 1-8 (2002).

[16] A. F. Sadreev and A. S. Pilipchuk, JETP Lett. 100, 585-590 (2014). 